\title{
Optical aurora detectors: using natural optics to motivate education and outreach
}

Joseph Shaw, Jesse Way, Nathan Pust, Paul Nugent, Hans Coate, et al.

Joseph A. Shaw, Jesse M. Way, Nathan J. Pust, Paul W. Nugent, Hans Coate, Daniel Balster, "Optical aurora detectors: using natural optics to motivate education and outreach," Proc. SPIE 9666, 11th Education and Training in Optics and Photonics Conference, 966608 (5 June 2009); doi:

$10.1117 / 12.2207940$

EDIE Event: Eleventh International Topical Meeting on Education and Training in Optics and Photonics, 2009, St. Asaph, United Kingdom 


\title{
Optical aurora detectors: using natural optics to motivate education and outreach
}

\author{
Joseph A. Shaw, ${ }^{\star}$ Jesse M. Way, Nathan J. Pust, \\ Paul W. Nugent, Hans Coate, Daniel Balster \\ Montana State University, Bozeman, Montana USA
}

\begin{abstract}
Natural optical phenomena enjoy a level of interest sufficiently high among a wide array of people to provide ideal education and outreach opportunities. The aurora promotes particularly high interest, perhaps because of its relative rarity in the areas of the world where most people live. A project is being conducted at Montana State University to use common interest and curiosity about auroras to motivate learning and outreach through the design and deployment of optical sensor systems that detect the presence of an auroral display and send cell phone messages to alert interested people. Project participants learn about the physics and optics of the aurora, basic principles of optical system design, radiometric calculations and calibrations, electro-optical detectors, electronics, embedded computer systems, and computer software. The project is moving into a stage where it will provide greatly expanded outreach and education opportunities as optical aurora detector kits are created and disbursed to colleges around our region.
\end{abstract}

Keywords: Aurora Borealis, aurora, atmospheric optics, optical sensors, optics education

\section{INTRODUCTION}

The aurora is a natural optical phenomenon that occurs primarily in high-latitude regions, created when energetic particles carried from the Sun by the solar wind collide with gas atoms and molecules in the upper levels of Earth's atmosphere, thereby releasing energy in the form of visible light. ${ }^{1,2}$ Its relative rarity at lower latitudes makes it a subject of significant curiosity, even in high-latitude regions and certainly in higher midlatitude regions where auroras are known to occur, but with relative rarity. In fact, the combination of knowing that an aurora can occur but not knowing when one might occur creates precisely the kind of interest that can be capitalized on to create effective opportunities for education and outreach.

When giving talks that include pictures of the Aurora Borealis (especially "local" pictures), the most common question, whether from university faculty or young school children, is something along the lines of how often an aurora can be seen where they live. Unfortunately, in nearly all cases, that question is essentially impossible to answer quantitatively. The answer certainly can begin with a geophysicist's map of aurora probability as a function of an observer's magnetic latitude and the 11-year sunspot cycle, ${ }^{1-3}$ but an assessment of how often those auroral displays might actually be seen also involves the probability of cloudiness for each location and the diligence of potential observers. The explanation usually goes on to address things like the $\mathrm{Ka}$ index and satellite-generated images of the auroral oval, ${ }^{4}$ which are all extremely useful indicators of what is happening now and what might happen in a few hours. Then the story often turns to describing the many late nights when a very active, expanded auroral oval have encouraged us to stay up late, only to see the oval retreat northward just as it is about to reach our location. Nevertheless, the story always includes pictures from nights when the reward for diligence was a display in the sky that ranges from a pale green glow on the northern horizon to a swirling red and green display dancing overhead.

A desire to eliminate missed aurora observing opportunities led to the idea of an automated way to detect when an aurora is occurring and notify ourselves and other interested persons of the viewing opportunity. In 1982 Knight $^{5}$ published a design for an optical aurora detector based on a photomultiplier tube (PMT) detector

* Contact author: jshaw@montana.edu 
and a 10-nm-wide interference filter centered on the $557.7 \mathrm{~nm}$ oxygen emission line that generates the most common green aurora. The PMT signal was detected and processed with analog circuitry that generated an audible alarm when an aurora was sensed. A chart recorder was used to record uncalibrated signal levels. In 1996, Haun $^{6}$ presented a digital upgrade to Knight's system using functionally identical optical components but upgrading the electronics to include a microprocessor for intelligent alarm detection. Alarm levels and dynamic gains were set in the microprocessor software and data could be recorded with a personal computer.

Recently, we developed an optical aurora detector that builds on these prior designs by adding a radiometric calibration to measure aurora brightness in addition to presence and to send out cell-phone text messages to a subscriber list of interested observers. This system uses an updated microcontroller and further reduces the amount of analog circuitry, but still relies on the same approach of detecting auroras with a PMT and 10-nmwide interference filter angle tuned so that the center of its bandpass is at $557.7 \mathrm{~nm}$. The prototype of this instrument was deployed at the Poker Flat Rocket Range (PFRR) ${ }^{7}$ near Fairbanks, Alaska, for testing and calibration during March 2007. These field tests verified the operation of the instrument and helped prepare it for continuous operation at our mid-latitude location in Bozeman, Montana $\left(45.67^{\circ} \mathrm{N}, 111.05^{\circ} \mathrm{W}\right)$.

\section{OPTICAL AURORA DETECTOR}

\subsection{System design and construction}

Figure 1 shows a block diagram layout of our prototype non-imaging optical aurora detector. In this figure, the four green blocks on the left are optical components, the blue center three blocks are analog electronic components, and the tan right four blocks are digital electronic components. Starting at the upper left corner of this diagram, light from the night sky is viewed through a 10-nm interference filter and 50-mm-diameter lens by a PMT. Rays traced from the outer edges of the PMT active area through the center of the lens determine the sensor's full-angle field of view (FOV). A trans-impedance amplifier conditions the analog signal, which is read on an analog input (analog-to-digital converter) to a microcontroller module, which allows the user to select a temporal averaging period to produce a smoothed signal. This signal is processed with a radiometric calibration and threshold algorithm that determines if an aurora is present and, if so, uses the absolute aurora radiance to generate a notification message that is sent as a text message to the cell phones of interested observers. A simple, unfiltered photodiode detector simultaneously observes the broadband sky brightness and provides a signal that can be used, if desired, to adjust the gain and thresholds of the aurora detector signal to account for variable moonlight and light pollution. We also use the photodiode signal to determine when to open and close a shutter that keeps the PMT dark during daytime.

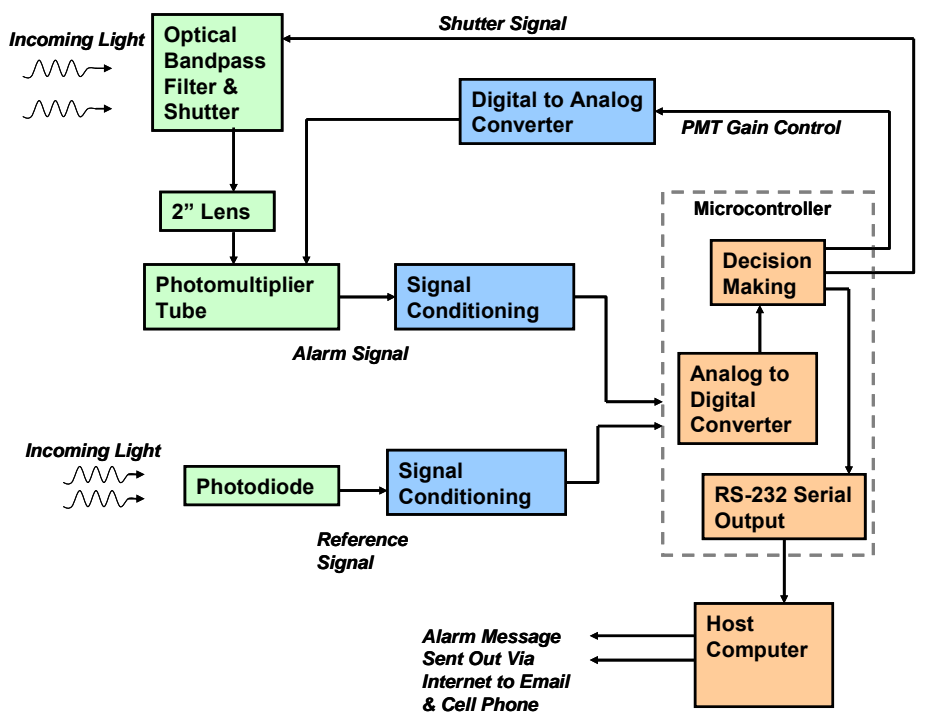

Fig. 1. Block diagram of the optical aurora detector system built at Montana State University in 2006-2007. 
The optical subsystem is illustrated in Fig. 2. An optical interference filter, with a full-width-at-half-maximum (FWHM) bandpass of $10 \mathrm{~nm}$, is tilted to tune its nominally 560-nm center wavelength to the oxygen emission line at $557.7 \mathrm{~nm}$. This makes use of the sometimes-useful and sometimes-annoying shift of the transmission peak of an interference filter to shorter wavelengths as the angle of the incident light is increased. Prior to constructing the filter mount, we measured the filter's transmission spectrum as a function of incidence angle and determined that a mounting surface tilted by $12^{\circ}$ would place our filter's transmission peak at $557.7 \mathrm{~nm}$.

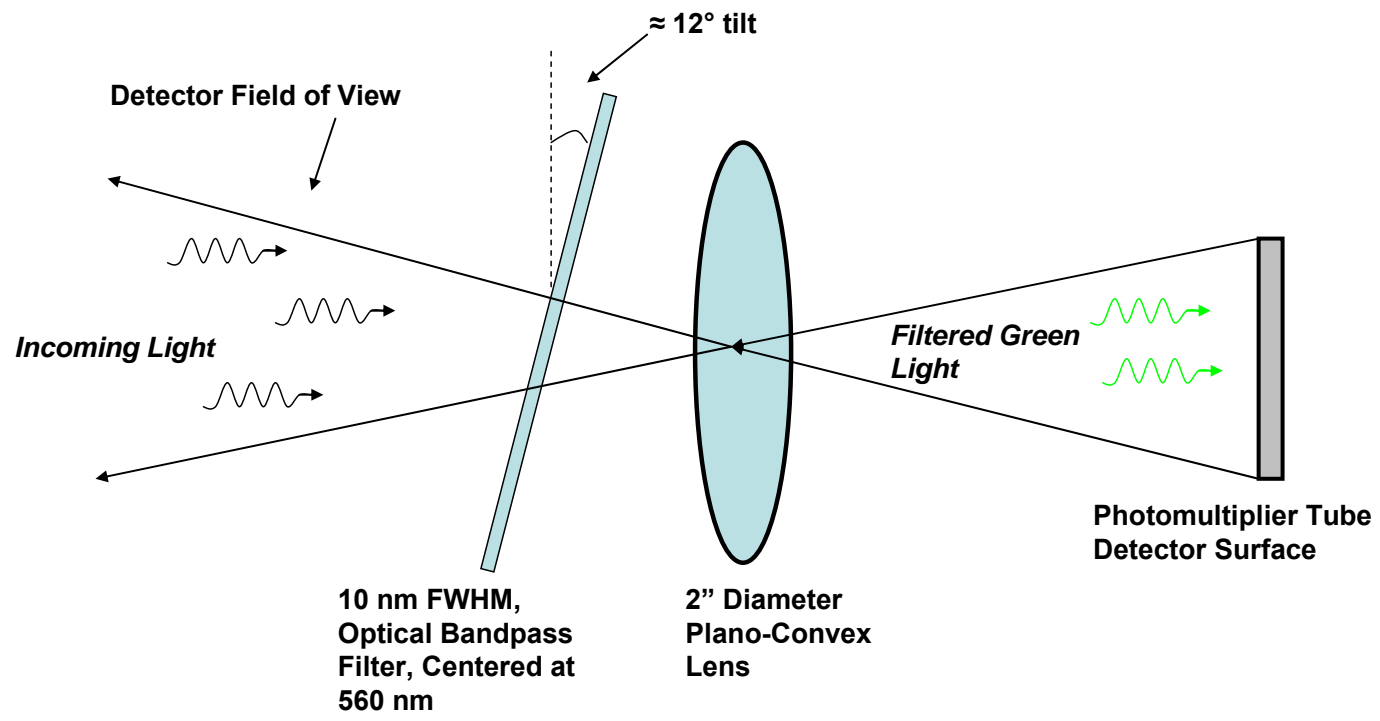

Fig. 2. Optical subsystem for the optical aurora detector. The interference filter is mounted at an angle that shifts the nominally 560-nm transmission peak to the oxygen emission wavelength of $557.7 \mathrm{~nm}$.

A photograph of the prototype system mounted in a weather-tight box is shown in Fig. 3 . The microcontroller and custom electronics board are mounted together on the left-hand side; the PMT module is at the lowerright, attached to a custom light-tight lens and filter mounting tube. At the top of the figure is a plexiglass window (clear) and custom shutter (black) that closes to maintain the PMT in darkness during daylight. The microcontroller signal is passed through a serial connection at the base of the box to a remote computer.

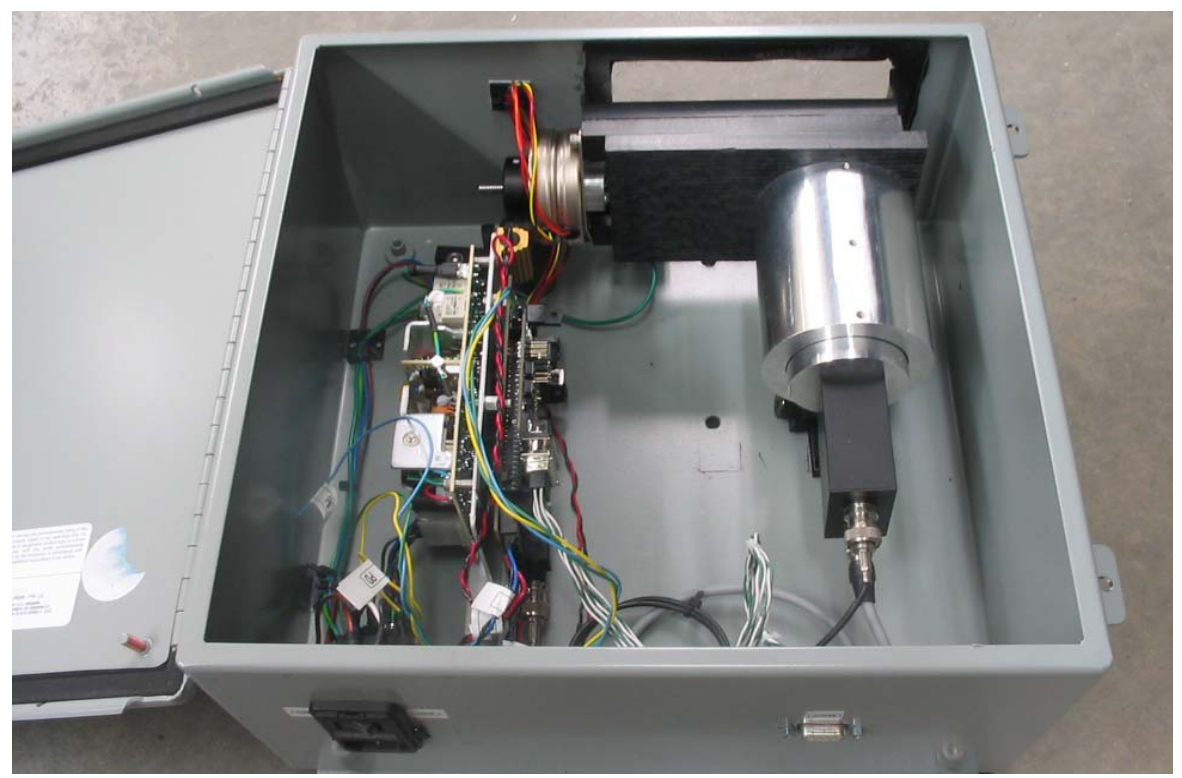

Fig. 3. Photograph of the optical aurora detector prototype. The microcontroller and electronics board are on the left-hand side; the PMT is the black module at the lower right, attached to a custom-machined light-tight lens and filter mount (silver tube, center right). A shutter (black module, top) opens to allow the PMT to view the sky at night. [J. Shaw photo]. 


\subsection{System testing in Alaska}

In March 2007 we took the prototype optical aurora detector to Alaska to be tested in an aurora-rich environment. Fig. 4 shows the system deployed in an observation dome at the Poker Flat Research Range (PFRR) operated by the University of Alaska near Fairbanks, Alaska (PFRR is at $65.13^{\circ} \mathrm{N}, 147.48^{\circ} \mathrm{W}$ ). During one week of testing, the system observed and detected auroras every night, ranging from just-visual wisps to bright, swirling displays.

Before deploying the system for aurora observations, we calibrated it using a standard lamp and barium sulfate reflecting screen. This calibration produced a curve relating output digital number to in-band radiance, which we convert to International Brightness Coefficient (IBC) units, which are used to classify aurora and airglow brightness in ranges from IBC1-IBC4, each separated by one decade. The IBC is determined in units of Rayleighs, ${ }^{8,9}$ which are defined at the reference wavelength of $557.7 \mathrm{~nm}$ as

$$
\text { 1Rayleigh }[R]=\frac{10^{10}}{4 \pi} \frac{\text { photons }}{\mathrm{s} \cdot \mathrm{m}^{2} \cdot \mathrm{sr}} \text {. }
$$

Table 1 lists the four IBC levels in Rayleighs, along with the corresponding radiance at $557.7 \mathrm{~nm}$ and a qualitative comparison to natural lighting situations. IBC I is subvisual to visible but below the onset of color vision, while IBC II is just beyond the human visual limit, which is only visible in dark skies with very low light pollution. IBC III and IBC IV correspond to bright, visually impressive displays. Fig. 5 is a photograph of the aurora near local midnight on 13 March 2007 at a time when the brightness approached IBC III. The photograph closely matches what was seen visually by observers at the PFRR. Fig. 6 is a time-series plot of the aurora detector signal during the nights of 13 March 2007.

Table 1. International Brightness Coefficient (IBC) levels and corresponding radiometric values

\begin{tabular}{|c|c|c|c|}
\hline IBC Level & Rayleighs & Radiance @ 557.7 nm & Relative Brightness \\
\hline I & $1 \mathrm{kR}$ & $2.833 \times 10^{-7} \mathrm{~W} /\left(\mathrm{m}^{2} \mathrm{sr}\right)$ & Appears white \\
\hline II & $10 \mathrm{kR}$ & $2.833 \times 10^{-6} \mathrm{~W} /\left(\mathrm{m}^{2} \mathrm{sr}\right)$ & $\begin{array}{c}\text { Onset of color } \\
\text { perception }\end{array}$ \\
\hline III & $100 \mathrm{kR}$ & $2.833 \times 10^{-5} \mathrm{~W} /\left(\mathrm{m}^{2} \mathrm{sr}\right)$ & Moonlit cumulus \\
\hline IV & $1000 \mathrm{kR}$ & $2.833 \times 10^{-4} \mathrm{~W} /\left(\mathrm{m}^{2} \mathrm{sr}\right)$ & Full moonlight \\
\hline
\end{tabular}

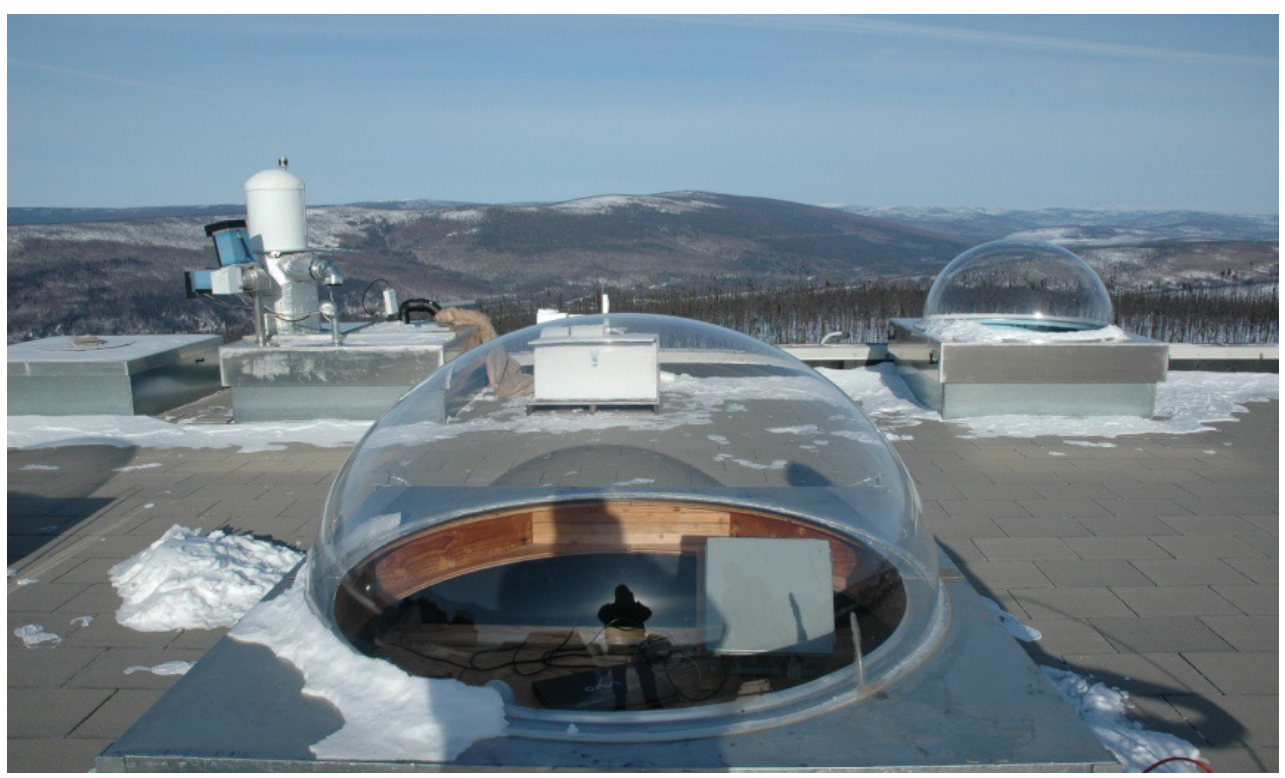

Fig. 4. Prototype optical aurora detector (gray box at lower right) deployed to view the northern horizon from a rooftop observation dome at PFRR near Fairbanks, Alaska for testing during March 2007. [J. Shaw photo]. 


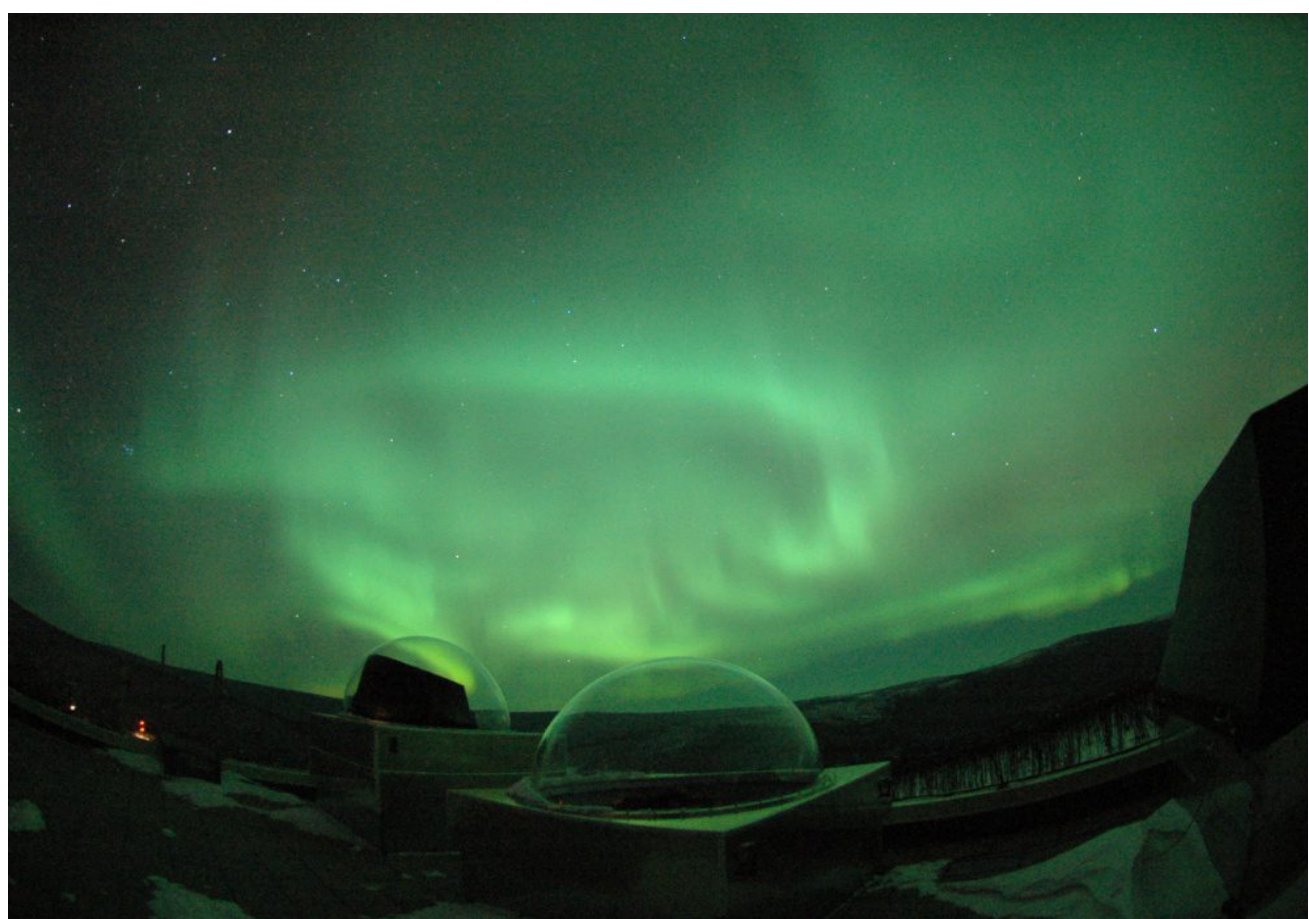

Fig. 5. Aurora observed near local midnight (UTC-9) on first night of testing in March 2007 [J. Shaw photo].

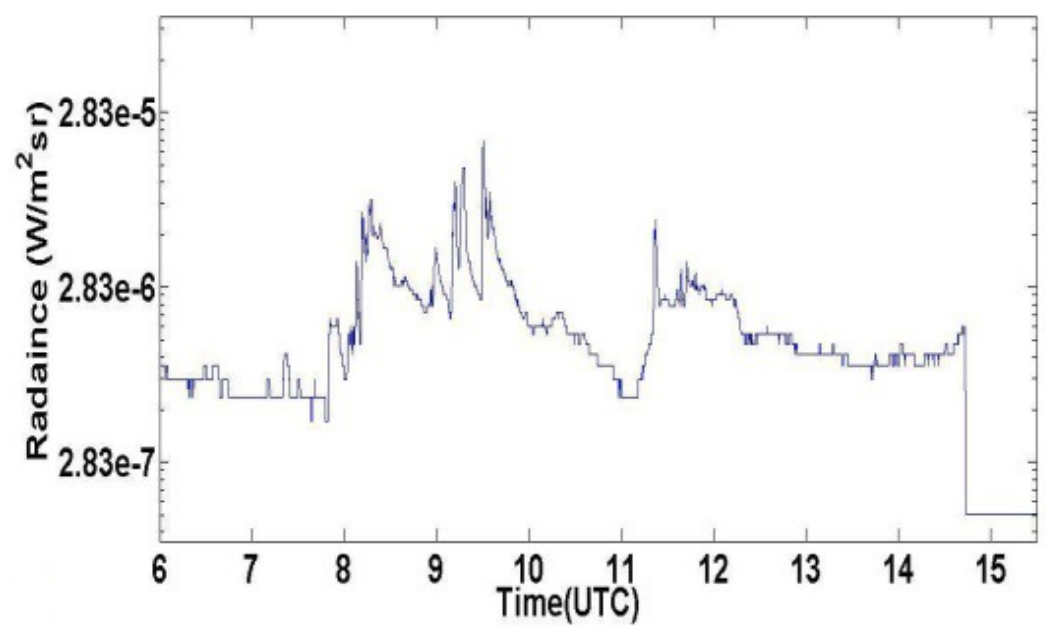

Fig. 6. Calibrated aurora detector signal measured on the night of 13 March 2007 at PFRR near Fairbanks, Alaska.

\section{Educational opportunities for students working on the aurora detectors}

A wide range of educational opportunities are provided in a natural, exciting manner for students in the process of designing, building, calibrating, and operating optical aurora detectors. For example, the topics learned by students working on this project include the following:

Basic physics of the Aurora

Radiometry and calculations of sensor signal-to-noise ratio

Radiometric sensor calibration

Aperture and field stops and geometric optics of radiometry

Interference filter angle tuning and designing field of view to match filter bandwidth

Mounting of optical components

Machining of custom optical mounts 
Detector parameters and selecting detector to meet specific signal-to-noise requirements

Amplifier circuits and electronic interfacing

Electronic circuit board layout and construction

Embedded microcontroller programming

Instrument thermal control

Light pollution and atmospheric scattering

In subsequent phases of this project, students have been exploring alternate instrument designs, including aurora imagers, and have been refining the thermal management and remote-control software options. Plans are being made to create student kits, so that students with not quite such sophisticated technical backgrounds can build and operate an instrument as part of an aurora detector network. Consequently, the range of opportunities to "learn by doing" has continued to expand. These developments will be discussed in more detail in future publications.

\section{Conclusion}

Building and operating optical aurora detectors is proving to be a highly motivational way for undergraduate engineering and science students to learn about topics ranging from optics to electronics to atmospheric physics to thermal control. In the course of their projects, students learn the required science or engineering subjects as they become necessary.

Following its successful Alaskan test, the original prototype sensor described here has been operating on the roof of the engineering building at Montana State University in Bozeman, Montana $\left(45.67^{\circ} \mathrm{N}, 111.05^{\circ} \mathrm{W}\right)$ for over two years. However, in that time no auroras have been available for detection owing to an unusually quiet period of low solar activity. As solar activity eventually increases during the upcoming cycle, the instrument will be ready to alert all interested people when an aurora can be seen in our area. Furthermore, the data we are continually collecting will provide unique insight into the statistics of the frequency of occurrence and absolute brightness of auroras in our area. As we develop a network of sensors, similar data will become available for different regions.

\section{Acknowledgments}

This is a Montana Space Grant project, funded through a NASA Space Grant Consortium education enhancement grant. We extend our deepest gratitude to the staff at the University of Alaska's Poker Flat Research Range for providing access to the range and assistance with our calibration measurements and instrument deployment in Alaska. Specifically, we acknowledge the assistance of Mr. Brian Lawson (Optics Engineer), Mr. Greg Walker (Range Manager), and Dr. Roger Smith (Director of the Geophysical Institute). We dedicate this paper to the memory of Dr. William Hiscock, former Director of the Montana Space Grant Consortium, whose support and excitement helped make this project possible.

\section{References}

[1] S. I. Akasofu, The Northern Lights: Secrets of the Aurora Borealis, Alaska Northwest Books, Reprint Edition (2009).

[2] N. Davis, Aurora watcher's handbook, University of Alaska Press (1992).

[3] J. A. Shaw, "What we see in the aurora," Optics and Photonics News 10(11), 20-24 (1999).

[4] Space Weather Prediction Center, www.swpc.noaa.gov, with current data and tutorials (2009).

[5] J. W. Knight, "Monitoring the aurora electronically," Sky and Telescope 63(6), 635-638 (1982).

[6] M. A. Haun, "The Aurora Alarm: an automated detection system for the Northern Lights," Walla Walla College, School of Engineering Seminar report, http://kolonia.keteu.org/ haunma/aurora/aurora.ps.gz (accessed July 2009).

[7] Poker Flat Research Range: www.pfrr.alaska.edu (2009).

[8] D. J. Baker, "Rayleigh, the Unit for Light Radiance," Appl. Opt. 13, 2160-2163 (1974).

[9] D. J. Baker and G. J. Romick, "The rayleigh: interpretation of the unit in terms of column emission rate or apparent radiance expressed in SI units," Appl. Opt. 15, 1966-1968 (1976). 